\title{
Sur les changements de signe d'une fonction harmonique dans le demi-plan
}

\author{
par \\ Lucien Chevalier et Alain Dufresnoy (Grenoble)
}

\begin{abstract}
In our recent paper [2], the study of the kernel associated with a singular integral led us to another question, relating to the boundary behaviour of the sign of a harmonic function in a half-plane. In this paper, the possible existence of sign oscillations of the Poisson integral $P(f)$ in the half-plane along rays is related to regularity properties of the boundary function $f$. This allows us to obtain a result of Fatou type for the sign of $P(f)$, under a regularity assumption that we prove to be optimal.
\end{abstract}

1. Introduction. Le problème étudié dans cet article trouve son origine dans une curieuse question de limite au bord pour le signe d'une fonction harmonique dans le demi-plan, que nous avons rencontrée récemment dans notre article [2], à propos de l'étude d'une certaine intégrale singulière.

Quelques notations sont nécessaires. Les espaces $L^{p}$ considérés sont relatifs à la mesure de Lebesgue $m$ dans $\mathbb{R}$, et la norme usuelle dans $L^{p}$ est notée $\|\cdot\|_{p}$. On désigne par $\mathbb{R}_{+}^{2}$ le demi-espace $\mathbb{R} \times \mathbb{R}_{+}$. Le point courant de $\mathbb{R}_{+}^{2}$ est systématiquement noté $z=(x, y)$, et le point courant $(\xi, 0)$ de la frontière de $\mathbb{R}_{+}^{2}$ est identifié avec le réel $\xi$. Pour tout point $\xi \in \mathbb{R}$, on note $p_{\xi}$ le noyau de Poisson relatif au point $\xi$, défini dans $\mathbb{R}_{+}^{2}$ par

$$
p_{\xi}(z)=\frac{1}{\pi} \cdot \frac{y}{(x-\xi)^{2}+y^{2}} .
$$

On désigne par $\mathcal{M}$ l'ensemble des applications mesurables de $\mathbb{R}$ dans $\mathbb{R}$ telles que

$$
\int_{\mathbb{R}} p_{\xi}(0,1)|f(\xi)| d \xi<+\infty .
$$

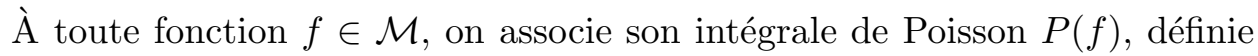
dans $\mathbb{R}_{+}^{2}$ par

$$
P(f)(z)=\int_{\mathbb{R}} p_{\xi}(z) f(\xi) d \xi
$$

2000 Mathematics Subject Classification: 31A05, 31A20. 
Avant d'énoncer notre résultat principal, résumons brièvement les raisons de notre intérêt pour la question étudiée dans le présent article. Soit $b$ une application borélienne bornée de $\mathbb{R}_{+}^{2}$ dans $\mathbb{R}$. Si on pose, pour toute fonction $f \in \mathcal{C}_{0}^{\infty}(\mathbb{R})$ et tout $\xi \in \mathbb{R}$,

$$
T_{b}(f)(\xi)=2 \int_{\mathbb{R}_{+}^{2}} y b(z) \nabla p_{\xi}(z) \nabla P(f)(z) d z,
$$

on définit un opérateur de Calderón-Zygmund $T_{b}$, associé au noyau $K_{b}$ défini par

$$
K_{b}\left(\xi, \xi^{\prime}\right)=2 \int_{\mathbb{R}_{+}^{2}} y b(z) \nabla p_{\xi}(z) \nabla p_{\xi^{\prime}}(z) d z
$$

pour $\xi \neq \xi^{\prime}$ ([1], proposition 3). En vue d'établir le résultat principal de [2], i. e. le fait que, pour toute fonction $f \in L^{2}$,

$$
\lim _{\varepsilon \rightarrow 0} \int_{\varepsilon<\left|\xi-\xi^{\prime}\right|} K_{\operatorname{sgn}(P(f))}\left(\xi, \xi^{\prime}\right) f\left(\xi^{\prime}\right) d \xi^{\prime}
$$

existe pour presque tout $\xi \in \mathbb{R}$, nous avons été conduits à nous demander plus généralement à quelle condition sur la fonction $b$,

$$
\lim _{\varepsilon \rightarrow 0} \int_{\varepsilon<\left|\xi-\xi^{\prime}\right|} K_{b}\left(\xi, \xi^{\prime}\right) f\left(\xi^{\prime}\right) d \xi^{\prime}
$$

existe pour presque tout $\xi \in \mathbb{R}$. Comme nous l'avons montré ([2], proposition 1), une condition nécessaire et suffisante pour que cette propriété ait lieu pour toute fonction $f \in L^{2}$ est que

$$
\lim _{\varepsilon \rightarrow 0} \int_{\mathbb{R}_{+}^{2}} b(\xi+\varepsilon z) \varrho(z) d z
$$

existe pour presque tout $\xi \in \mathbb{R}$, où

$$
\varrho(z)=\frac{4}{\pi^{2}} \cdot \frac{y\left(y^{4}+x^{4}+2 y^{2} x^{2}-x^{2}+y^{2}\right)}{\left(x^{2}+y^{2}\right)^{2}\left((x-1)^{2}+y^{2}\right)\left((x+1)^{2}+y^{2}\right)} .
$$

Ce résultat suscite immédiatement la question suivante : étant donné une fonction $f \in L^{2}$, est-il vrai que la fonction $b=\operatorname{sgn}(P(f))$ possède la propriété précédente? À défaut, quelle propriété supplémentaire doit posséder la fonction $f$ pour qu'il en soit ainsi ?

Comme nous allons le voir, la réponse à la première partie de la question est négative en général, mais positive pour des fonctions $f$ suffisamment régulières. Ceci explique pourquoi nous avons cherché à déterminer avec précision le degré de régularité requis pour que la propriété soit vraie. Par ailleurs, le fait qu'une fonction quelconque de $L^{2}$ ne vérifie pas la propriété explique aussi pourquoi la preuve du résultat principal de [2] a nécessité une analyse plus précise que la simple application de la théorie de CalderónZygmund-Cotlar. 
Cette question suscite plusieurs remarques, qui permettront de mieux cerner le problème; pour cela, nous introduisons deux définitions. Étant donné une fonction borélienne bornée $b$ définie dans le demi-espace et un point $\xi \in \mathbb{R}$, nous dirons que cette fonction vérifie la propriété $L^{\prime}(\xi)$ si, pour presque tout $z \in \mathbb{R}_{+}^{2}$ (relativement à la mesure de Lebesgue plane),

$$
\lim _{\varepsilon \rightarrow 0} b(\xi+\varepsilon z) \quad \text { existe. }
$$

D'autre part, étant donné une mesure (borélienne, signée, à variation totale finie) $\mu$ dans $\mathbb{R}_{+}^{2}$, nous dirons que la fonction $b$ vérifie la propriété $L_{\mu}^{\prime \prime}(\xi)$ si

$$
\lim _{\varepsilon \rightarrow 0} \int_{\mathbb{R}_{+}^{2}} b(\xi+\varepsilon z) \mu(d z) \quad \text { existe. }
$$

La question posée plus haut est donc celle de savoir à quelle condition la fonction $\operatorname{sgn}(P(f))$ possède la propriété $L_{\mu}^{\prime \prime}(\xi)$ pour presque tout $\xi \in \mathbb{R}$, lorsqu'on prend $\mu(d z)=\varrho(z) d z$ (il est facile de vérifier que la fonction $\varrho$ est intégrable dans $\mathbb{R}_{+}^{2}$ par rapport à la mesure de Lebesgue plane).

Entre les deux propriétés de convergence $L^{\prime}$ et $L^{\prime \prime}$ existent des relations évidentes : il est clair que $L^{\prime}(\xi)$ implique $L_{\mu}^{\prime \prime}(\xi)$ pour toute mesure $\mu$ absolument continue par rapport à la mesure de Lebesgue plane ; inversement, la propriété $L_{\mu}^{\prime \prime}(\xi)$ pour toute mesure $\mu$ implique évidemment $L^{\prime}(\xi)$. Par ailleurs, à moins qu'une mesure $\mu$ soit douée de propriétés particulières vis à vis de la fonction $b$ (ce qui ne semble pas, d'après nos résultats, être le cas dans la situation qui nous intéresse ici), la seule tactique réaliste pour établir $L_{\mu}^{\prime \prime}(\xi)$ dans le cas d'une mesure $\mu$ absolument continue nous semble être de prouver $L^{\prime}(\xi)$.

Notons enfin que, dans le cas où la fonction $b$ est de la forme $\operatorname{sgn}(P(f))$, elle vérifie automatiquement la propriété $L^{\prime}(\xi)$, en presque tout point $\xi \in$ $\mathbb{R}$ tel que $f(\xi) \neq 0$. En effet, d'après le théorème de Fatou, on sait que $P(f)$ admet, en presque tout point $\xi$ de la frontière de $\mathbb{R}_{+}^{2}$, une limite non tangentielle égale à $f(\xi)$. Par conséquent, la fonction $\operatorname{sgn}(P(f))$ possède la propriété $L^{\prime}(\xi)$ en presque tout point $\xi \in \mathbb{R}$ si et seulement si, en presque tout $\xi$ tel que $f(\xi)=0$, la fonction harmonique $P(f)$ n'a qu'un nombre fini de changements de signe sur la demi-droite issue du point $\xi$ et passant par $z$, pour presque tout $z \in \mathbb{R}_{+}^{2}$.

Pour énoncer commodément le résultat principal de cet article, nous introduisons la notation suivante : pour toute application $f: \mathbb{R} \rightarrow \mathbb{R}$, et tout $r>0$, nous poserons

$$
\sigma_{f}(r)=\sup _{x \in \mathbb{R} ;|h| \leq r}|f(x+h)-f(x)| .
$$

Le résultat suivant indique avec précision, en termes de module de continuité, quelle propriété de régularité doit posséder une fonction pour que le 
signe de son extension harmonique vérifie la propriété $L^{\prime}(\xi)$ en presque tout point $\xi \in \mathbb{R}$.

THÉORÈmE. Il y a, entre la régularité d'une fonction et le comportement à la frontière du signe de son intégrale de Poisson, les relations suivantes:

(i) Pour toute fonction $f \in \mathcal{M}$ telle que la fonction $r \mapsto \sigma_{f}(r) / r$ soit bornée (ce qui revient à dire que $f \in \operatorname{Lip}_{1}(\mathbb{R})$ ), la fonction $\operatorname{sgn}(P(f))$ vérifie la propriété $L^{\prime}(\xi)$ pour presque tout $\xi \in \mathbb{R}$.

(ii) Pour toute application croissante $\sigma:[0,+\infty[\rightarrow[0,+\infty[$, vérifiant $\sigma(0)=0$ et telle que $\sigma(r) / r \rightarrow+\infty$ quand $r \rightarrow 0$, il existe une fonction bornée $g$ définie dans $\mathbb{R}$, vérifiant $\sigma_{g} \leq \sigma$, et un compact $L$ de mesure de Lebesgue $>0$, possédant les propriétés suivantes :

- Pour tout $\xi \in L$, la restriction à toute droite issue de $\xi$ de la fonction $\operatorname{sgn}(P(g))$ n'admet pas de limite au point $\xi$.

- Pour tout $\xi \in L$, et toute mesure borélienne $\mu$ sur $\mathbb{R}_{+}^{2}$, à valeurs réelles et à variation totale finie, telle que $\mu\left(\mathbb{R}_{+}^{2}\right) \neq 0$, l'intégrale

$$
\int_{\mathbb{R}_{+}^{2}} \operatorname{sgn}(P(g)(\xi+\varepsilon z)) \mu(d z)
$$

n'a pas de limite quand $\varepsilon$ tend vers 0 .

Observons que l'assertion (ii) implique notamment l'existence d'une fonction bornée $f \in \bigcap_{0<\alpha<1} \operatorname{Lip}_{\alpha}$ telle que la fonction $\operatorname{sgn}(P(f))$ ne vérifie pas la propriété $L^{\prime}(\xi)$ pour tous les points $\xi$ d'un ensemble de mesure positive. D'autre part, on peut montrer $([2])$ que $\int_{\mathbb{R}_{+}^{2}} \varrho(z) d z=1$, donc la deuxième partie de l'assertion (ii) donne le résultat annoncé plus haut, dans le cas où $\mu(d z)=\varrho(z) d z$.

\section{Démonstration du théorème}

2.1. Démonstration de l'assertion (i). Nous commençons par exclure le cas trivial dans lequel la fonction $f$ est constante. Dans le cas contraire, la première étape consiste à prouver que $\nabla P(f)$ a, en presque tout point de $\mathbb{R}$, une limite non-tangentielle non nulle. Par hypothèse, la fonction $f$ est dérivable presque partout, sa dérivée $f^{\prime}$ est bornée et $\partial P(f) / \partial x=P\left(f^{\prime}\right)$. Si $H$ désigne la transformation de Hilbert sur la droite, la fonction

$$
(x+i y) \mapsto F(x+i y)=P\left(f^{\prime}\right)(x, y)+i P\left(H f^{\prime}\right)(x, y)
$$

est holomorphe dans le demi-plan. Elle vérifie l'égalité $|\nabla P(f)(x, y)|=$ $|F(x+i y)|$ pour tout $(x, y) \in \mathbb{R}_{+}^{2}$ et admet une limite non-tangentielle presque partout dans $\mathbb{R}$, parce que $f^{\prime}$ est bornée. De plus, cette limite nontangentielle ne peut s'annuler sur un ensemble de mesure $>0$, car sinon la fonction holomorphe bornée $G=e^{F}-1$ aurait une limite non-tangentielle nulle sur cet ensemble, donc serait identiquement nulle. Par suite $F$ serait 
nulle et $f$ serait constante, ce que nous avons exclu. On en conclut donc que $\nabla P(f)$ a, en presque tout point de $\mathbb{R}$, une limite non-tangentielle non nulle.

Pour achever la démonstration, il suffit donc de vérifier que, si un point $\xi \in \mathbb{R}$ est tel que la limite non-tangentielle $G(\xi)$ de $\nabla P(f)$ en ce point soit non nulle, alors la fonction $\operatorname{sgn}(P(f))$ vérifie la propriété $L^{\prime}(\xi)$. Or, pour que la restriction de $\operatorname{sgn}(P(f))$ à une demi-droite issue de $\xi$ n'ait pas de limite en $\xi$, il est nécessaire que $P(f)$ s'annule une infinité de fois sur cette demi-droite, et dans ce cas le théorème de Rolle permet de voir que $G(\xi)$ est orthogonal à cette demi-droite. Comme $G(\xi) \neq 0$, cela ne peut arriver que pour une demi-droite issue de $\xi$ au plus, donc la propriété voulue est établie.

2.2. Démonstration de l'assertion (ii). Pour des raisons techniques, nous commencerons par construire une fonction $f$ définie dans le cercle unité du plan complexe et un compact $K$ inclus dans ce cercle unité, qui possèdent des propriétés similaires à celles que devront vérifier la fonction $g$ définie sur $\mathbb{R}$ et le compact $L$. Nous obtiendrons ensuite $g$ et $L$ à partir de $f$ et $K$ au moyen d'un argument de représentation conforme.

Préliminaires. Nous partons de l'ensemble $\Omega^{\prime}$ des points $z \in \mathbb{C}$ tels que $0<\Re z<1$ et $|\Im z|<1$; nous "arrondissons les angles" de façon à ce que sa frontière devienne de classe $\mathcal{C}^{2}$, et nous notons $\Omega$ le nouvel ouvert ainsi régularisé. Pour tout $z \in \mathbb{C}$ et tout $r>0$, nous notons $D(z, r)$ le disque ouvert de centre $z$ et de rayon $r$, et $D=D(0,1)$ le disque unité. Pour tout $\alpha \in] 0,1\left[\right.$, nous désignons par $\varphi_{\alpha}$ la représentation conforme de $D$ sur $\Omega$ telle que $\varphi_{\alpha}(0)=\alpha$ et telle que $\varphi_{\alpha}^{\prime}(0)$ soit un réel $>0$. L'application $\varphi_{\alpha}$ se prolonge à $\bar{D}$ en une application de classe $\mathcal{C}^{1}$, que nous noterons encore $\varphi_{\alpha}$.

L'ensemble $N_{\alpha}$ des points $\xi \in \partial D$ tels que $\Re \varphi_{\alpha}(\xi)=0$ est un intervalle du cercle $\partial D$, et sa mesure de Lebesgue $\left(^{1}\right)\left|N_{\alpha}\right|$ tend vers $2 \pi$ quand $\alpha$ tend vers 0 . En effet, on a $\left|N_{\alpha}\right|=\mu_{\alpha}(A)$, où $\mu_{\alpha}$ est la mesure image par $\varphi_{\alpha}$ de la mesure de Lebesgue sur $\partial D$, et $A$ est l'ensemble des points $z \in \partial \Omega$ tels que $\Re z=0$. Comme l'application $\varphi_{\alpha}$ transforme la mesure harmonique relative au point 0 de $D$ (i. e. la mesure de Lebesgue normalisée sur $\partial D$ ) en la mesure harmonique relative au point $\alpha$ de $\Omega$, on voit que $\left|N_{\alpha}\right| /(2 \pi)$ est la valeur au point $\alpha$ de l'extension harmonique à $\Omega$ de la fonction indicatrice de $A$, et il est clair que cette valeur tend vers 1 lorsque $\alpha$ tend vers 0 .

$\mathrm{Au}$ voisinage de tout point $\xi \in \stackrel{\circ}{N}_{\alpha}$, la fonction $\varphi_{\alpha}$ se prolonge en une fonction holomorphe $\varphi$ (il s'agit essentiellement d'une application du principe de symétrie de Schwarz); de plus $\varphi^{\prime}(\xi) \neq 0$ et $\varphi^{\prime}(\xi) / \xi \in \mathbb{R}$ (parce que la transformation $\varphi_{\alpha}$ est conforme).

Pour tout $\xi \in \partial D$ et tout $b \in] 0,1\left[\right.$, on désigne par $\Gamma_{b}(\xi)$ l'enveloppe convexe de l'ensemble $D(0, b) \cup\{\xi\}$. Ce qui précède permet de montrer

$\left({ }^{1}\right)$ Il s'agit bien entendu de la mesure longueur sur le cercle. 
facilement que, pour tout compact $K \subset \stackrel{\circ}{N}_{\alpha}$ et tout $\left.b \in\right] 0,1[$, il existe $C(\alpha, K, b)>0$ et $\varrho(\alpha, K, b)>0$ tels que, pour tout $\xi \in K$ et tout $z \in \Gamma_{b}(\xi)$ vérifiant $|z-\xi| \leq \varrho(\alpha, K, b)$, on ait

$$
\Re \varphi_{\alpha}(z) \geq C(\alpha, K, b)|z-\xi| .
$$

D'autre part, pour tout entier $k \geq 1$ et tout $b \in] 0,1$ [, il existe un nombre $\eta(k, b)>0$ tel que, pour tout $\xi \in \partial D$,

$$
p_{k}\left(\Gamma_{b}(\xi) \cap D(\xi, \eta(k, b))\right) \subset \Gamma_{(1+b) / 2}\left(p_{k}(\xi)\right),
$$

où $p_{k}$ désigne l'application $z \mapsto z^{k}$. De ces deux propriétés, on déduit que, pour tout compact $K \subset \stackrel{\circ}{N}_{\alpha}$, tout entier $k \geq 1$ et tout $\left.b \in\right] 0,1[$, il existe un nombre $\varrho(\alpha, K, k, b)>0$ tel que, pour tout $\xi \in p_{k}^{-1}(K)$ et tout $z \in \Gamma_{b}(\xi)$ vérifiant $|z-\xi| \leq \varrho(\alpha, K, k, b)$, on ait

$$
\Re \varphi_{\alpha}\left(z^{k}\right) \geq \frac{k}{2} C(\alpha, K,(b+1) / 2)|z-\xi| .
$$

Remarquons (ce qui nous sera utile dans la suite) que les constantes $C(\alpha, K, b)$ et $\varrho(\alpha, K, k, b)$ conviennent a fortiori si on remplace le compact $K$ par un sous-ensemble $K^{\prime}$ de $K$.

Construction de la fonction $f$. On se propose de construire la fonction pathologique $f$ sous la forme

$$
\xi \mapsto \sum_{n=1}^{+\infty}(-1)^{n} a_{n} \Re \varphi_{\alpha_{n}}\left(\xi^{k_{n}}\right),
$$

où les suites $\left(a_{n}\right),\left(\alpha_{n}\right)$ et $\left(k_{n}\right)$ sont convenablement choisies. On choisit tout d'abord pour $\left(\alpha_{n}\right)$ une suite décroissante de points de ]0, 1[ telle que le produit infini

$$
\prod_{n=1}^{+\infty}\left(1-2\left(1-\frac{\left|N_{\alpha_{n}}\right|}{2 \pi}\right)\right)
$$

soit convergent. D'autre part, on se donne une suite arbitraire $\left(b_{n}\right)$ de points de $] 0,1[$ qui converge vers 1 .

Il reste donc à construire les suites $\left(a_{n}\right)$ et $\left(k_{n}\right)$, ainsi que le compact $K$. Pour effectuer cette construction, nous utiliserons le

Lemme 1. Il existe une suite $\left(K_{n}\right)$ de parties compactes de $\partial D$, une suite $\left(k_{n}\right)$ d'entiers, et trois suites $\left(a_{n}\right),\left(c_{n}\right)$ et $\left(\varrho_{n}\right)$ de nombres réels telles qu'on ait, pour tout entier $n \geq 1$, les propriétés suivantes :

$\left(1_{n}\right) \quad K_{n}$ est une union finie d'intervalles fermés de mesure $>0$ et $K_{n} \subset$ $p_{k_{n}}^{-1}\left({\stackrel{\circ}{\alpha_{n}}}\right)$. De plus, pour $2 \leq p \leq n, K_{p} \subset K_{p-1}$ et

$$
\left|K_{p}\right| \geq\left(1-2\left(1-\frac{\left|N_{\alpha_{p}}\right|}{2 \pi}\right)\right)\left|K_{p-1}\right| .
$$


$\left(2_{n}\right) \quad c_{1} \leq \ldots \leq c_{n}$ et $c_{n} \geq n$ pour $n \geq 2$.

$\left(3_{n}\right) \quad \varrho_{p} \leq 2^{-(p-1)} \varrho_{p-1}$ pour $2 \leq p \leq n$ et $c_{n} \varrho_{n} \leq c_{n-1} \varrho_{n-1} \leq \ldots \leq c_{1} \varrho_{1}$.

$\left(4_{n}\right) \quad a_{n} \leq a_{n-1} \leq \ldots \leq a_{1}$ et $a_{p} \leq \min \left(2^{-p-1} c_{p-1} \varrho_{p-1}, 2^{-p+1} a_{p-1}\right)$ pour $2 \leq p \leq n$

$\left(5_{n}\right) \quad$ Pour tout $m \in\{1, \ldots, n\}$, tout $\xi \in K_{m}$ et tout $z \in \Gamma_{b_{m}}(\xi)$ tel que $2^{-m} \varrho_{m} \leq|z-\xi| \leq \varrho_{m}$

$$
\left|\sum_{p=1}^{m}(-1)^{p} a_{p} \Re \varphi_{\alpha_{p}}\left(z^{k_{p}}\right)\right| \geq \frac{c_{m} \varrho_{m}}{2^{m}}
$$

et $\sum_{p=1}^{m}(-1)^{p} a_{p} \Re \varphi_{\alpha_{p}}\left(z^{k_{p}}\right)$ est du signe de $(-1)^{m}$.

$\left(6_{n}\right) \quad$ Pour tout $m \in\{1, \ldots, n\}$,

$$
\sum_{p=1}^{m} a_{p} k_{p}\left\|\varphi_{\alpha_{p}}^{\prime}\right\|_{\infty}+2 \leq \tau\left(2 a_{m}\right),
$$

où $\left\|\varphi_{\alpha_{p}}^{\prime}\right\|_{\infty}=\sup _{z \in D}\left|\varphi_{\alpha_{p}}^{\prime}(z)\right|$ et la fonction $\tau$ est définie par $\tau(t)=$ $\inf _{h \leq t} \sigma(h / 2) / h$.

Démonstration. Les suites seront construites de proche en proche. Nous utiliserons nos remarques préliminaires, en conservant les notations introduites à cette occasion. Pour la première étape, on choisit arbitrairement $k_{1}=1$, et on prend pour $K_{1}$ un intervalle fermé inclus dans ${\stackrel{\circ}{\alpha_{1}}}_{1}$ tel que $\left|K_{1}\right|>0$; la condition $\left(1_{1}\right)$ est ainsi satisfaite. On choisit ensuite pour $a_{1}$ un nombre $>0$ assez petit pour que la condition $\left(6_{1}\right)$ soit satisfaite, ce qui est rendu possible par le fait que $\tau(u)$ tend vers $+\infty$ quand $u$ tend vers 0 . Enfin, d'après nos remarques préliminaires (inégalité (3)), il suffit de prendre $c_{1}=a_{1} C\left(\alpha_{1}, K_{1}, b_{1}\right)$ et $\varrho_{1}=\varrho\left(\alpha_{1}, K_{1}, b_{1}\right)$ pour que la propriété $\left(5_{1}\right)$ soit satisfaite. Ceci termine la première étape de la démonstration, les conditions $\left(2_{1}\right),\left(3_{1}\right)$ et $\left(4_{1}\right)$ étant vides.

Supposons maintenant construites des séquences

$$
\left(K_{1}, \ldots, K_{n}\right), \quad\left(k_{1}, \ldots, k_{n}\right), \ldots,\left(\varrho_{1}, \ldots, \varrho_{n}\right)
$$

vérifiant les conditions $\left(1_{n}\right)$ à $\left(6_{n}\right)$, et montrons qu'il est possible de construire $K_{n+1}, k_{n+1}, \ldots, \varrho_{n+1}$ de façon à ce que les séquences

$$
\left(K_{1}, \ldots, K_{n+1}\right), \quad\left(k_{1}, \ldots, k_{n+1}\right), \ldots,\left(\varrho_{1}, \ldots, \varrho_{n+1}\right)
$$

vérifient les conditions $\left(1_{n+1}\right)$ à $\left(6_{n+1}\right)$.

Pour cela, nous commençons par remarquer la propriété élémentaire suivante : pour tout couple $(I, J)$ d'intervalles de $\partial D,\left|I \cap p_{k}^{-1}(J)\right|$ tend vers $|I| \cdot|J| /(2 \pi)$ quand $k$ tend vers l'infini. En appliquant cette propriété à toute composante connexe $I$ de $K_{n}$ et en utilisant la régularité de la mesure de Lebesgue, on en déduit l'existence d'un entier $p$ tel que, pour tout entier $k \geq p$, il existe une union finie $L_{k}$ d'intervalles fermés de $\partial D$ possédant les 
propriétés suivantes :

$$
L_{k} \subset K_{n} \cap p_{k}^{-1}\left(\stackrel{\circ}{N}_{\alpha_{n+1}}\right)
$$

et

$$
\left|L_{k}\right|=\left|K_{n} \cap L_{k}\right| \geq\left(1-2\left(1-\frac{\left|N_{\alpha_{n+1}}\right|}{2 \pi}\right)\right)\left|K_{n}\right| .
$$

Nous choisirons ultérieurement pour $k_{n+1}$ un entier $\geq p$, puis nous complèterons la séquence $\left(K_{1}, \ldots, K_{n}\right)$ en posant $K_{n+1}=L_{k_{n+1}}$. La condition $\left(1_{n+1}\right)$ sera ainsi vérifiée.

Par ailleurs, si nous complétons la séquence $\left(c_{1}, \ldots, c_{n}\right)$ en posant

$$
c_{n+1}=\max \left(c_{n}, n+1\right),
$$

la condition $\left(2_{n+1}\right)$ est remplie.

D'autre part, si $a_{n+1}$ et $k_{n+1}$ sont choisis de façon à ce que leur produit vérifie

$$
\frac{a_{n+1} k_{n+1}}{2} C\left(\alpha_{n+1}, K_{n},\left(1+b_{n+1}\right) / 2\right)-\sum_{p=1}^{n} a_{p} k_{p}\left\|\varphi_{\alpha_{p}}^{\prime}\right\|_{\infty} \geq \max \left(c_{n}, n+1\right),
$$

on a, pour tout compact $L \subset K_{n}$, tout $\xi \in L$ et tout $z \in \Gamma_{b_{n+1}}(\xi)$ tel que $|z-\xi| \leq \varrho\left(\alpha_{n+1}, K_{n}, k_{n+1}, b_{n+1}\right)$, les inégalités

$$
\begin{aligned}
& \left|\sum_{p=1}^{n+1}(-1)^{p} a_{p} \Re \varphi_{\alpha_{p}}\left(z^{k_{p}}\right)\right| \\
& \quad \geq a_{n+1} \Re \varphi_{\alpha_{n+1}}\left(z^{k_{n+1}}\right)-\left(\sum_{p=1}^{n} a_{p} k_{p}\left\|\varphi_{\alpha_{p}}^{\prime}\right\|_{\infty}\right)|z-\xi| \geq c_{n+1}|z-\xi|,
\end{aligned}
$$

en vertu de l'inégalité (4) et du choix de $c_{n+1}$.

Nous choisissons maintenant un entier $P_{n+1}$ (destiné à être le futur produit $\left.a_{n+1} k_{n+1}\right)$ vérifiant l'inégalité

$$
P_{n+1} \geq \frac{2\left(\max \left(c_{n}, n\right)+\sum_{p=1}^{n} a_{p} k_{p}\left\|\varphi_{\alpha_{p}}^{\prime}\right\|_{\infty}\right)}{C\left(\alpha_{n+1}, K_{n}, b_{n+1}\right)} .
$$

Ce nombre étant fixé, les propriétés de la fonction $\tau$ nous permettent de choisir un nombre $\delta>0$ tel que, pour tout $a \leq \delta$, on ait

$$
P_{n+1}\left\|\varphi_{\alpha_{n+1}}^{\prime}\right\|_{\infty}+\sum_{p=1}^{n} a_{p} k_{p}\left\|\varphi_{\alpha_{p}}^{\prime}\right\|_{\infty}+2 \leq \tau(2 a) .
$$

Nous prenons alors pour $a_{n+1}$ un nombre $>0$ tel que $1 / a_{n+1}$ soit entier, et vérifiant l'inégalité

$$
a_{n+1} \leq \min \left(\frac{c_{n} \varrho_{n}}{2^{n+1}}, \frac{a_{n}}{2^{n}}, \delta, \frac{P_{n+1}}{p}\right),
$$


puis nous posons $k_{n+1}=P_{n+1} / a_{n+1}$. La séquence $a_{1}, \ldots, a_{n+1}$ vérifie trivialement $\left(4_{n+1}\right)$, et il résulte de l'inégalité $(6)$ que la condition $\left(6_{n+1}\right)$ est également satisfaite.

Nous choisissons maintenant un nombre $\varrho_{n+1}$ vérifiant

$$
0<\varrho_{n+1} \leq \min \left(2^{-n} \varrho_{n}, \frac{c_{n}}{c_{n+1}} \varrho_{n}, \varrho\left(\alpha_{n+1}, K_{n}, k_{n+1}, b_{n+1}\right)\right) .
$$

La condition $\left(3_{n+1}\right)$ est alors satisfaite. De plus, compte tenu de ce choix et de l'inclusion $K_{n+1} \subset K_{n}$, les inégalités (5) nous font voir que, pour tout $\xi \in K_{n+1}$ et tout $z \in \Gamma_{b_{n+1}}(\xi)$ tel que $2^{-(n+1)} \varrho_{n+1} \leq|z-\xi| \leq \varrho_{n+1}$,

$$
\left|\sum_{p=1}^{n+1}(-1)^{p} a_{p} \Re \varphi_{\alpha_{p}}\left(z^{k_{p}}\right)\right| \geq \frac{c_{n+1} \varrho_{n+1}}{2^{n+1}}
$$

et $\sum_{p=1}^{n+1}(-1)^{p} a_{p} \Re \varphi_{\alpha_{p}}\left(z^{k_{p}}\right)$ est du signe de $(-1)^{n+1}$. La condition $\left(5_{n+1}\right)$ étant ainsi vérifiée, la démonstration du lemme est achevée et nous pouvons revenir à la preuve du théorème.

Nous conservons les notations précédentes. Les suites $\left(K_{n}\right), \ldots,\left(\varrho_{n}\right)$ étant construites, nous posons, pour tout entier $n \geq 1$ et tout $z \in \bar{D}$,

$$
u_{n}(z)=\lambda \sum_{p=1}^{n}(-1)^{p} a_{p} \Re \varphi_{\alpha_{p}}\left(z^{k_{p}}\right),
$$

où $\lambda$ est une constante vérifiant $0<\lambda \leq 1$, et qui sera ajustée à la fin de la démonstration. Les inégalités $\left(4_{n}\right)$ ont comme conséquence le fait que la suite $\left(u_{n}\right)$ converge uniformément dans $\bar{D}$ vers une fonction $u$ continue dans $\bar{D}$ et harmonique dans $D$. Il est donc clair que la restriction de $u$ à $D$ n'est autre que $P(f)$, où $f$ est la fonction définie dans $\partial D$ par l'égalité

$$
f(\xi)=\lambda \sum_{n=1}^{+\infty}(-1)^{n} a_{n} \Re \varphi_{\alpha_{n}}\left(\xi^{k_{n}}\right) .
$$

Preuve des propriétés de la fonction $f$. Nous commencerons par construire un compact $K$ (qui aura des propriétés similaires à celles qui sont demandées à $L$ dans l'énoncé) en posant

$$
K=\bigcap_{n=1}^{+\infty} K_{n}
$$

Comme la suite $\left(K_{n}\right)$ est décroissante, on a $|K|=\lim _{n \rightarrow+\infty}\left|K_{n}\right|$, donc il résulte des conditions $\left(1_{n}\right)$ et du choix de la suite $\left(\alpha_{n}\right)$ que $|K|>0$. Étant donné un point $\xi \in K$ et un entier $n \geq 1$, nous désignerons dans la suite par $\Omega_{n}(\xi)$ l'ensemble des points $z \in \Gamma_{b_{n}}(\xi)$ tels que $2^{-n} \varrho_{n} \leq|z-\xi| \leq \varrho_{n}$.

Nous allons maintenant montrer que, pour tout point $\xi \in K$ et tout entier $n \geq 1, P(f)(z)(=u(z))$ est du signe de $(-1)^{n}$ dans l'ensemble $\Omega_{n}(\xi)$. 
Pour cela il suffit, en vertu de $\left(5_{n}\right)$, de montrer que $u(z)$ et $u_{n}(z)$ sont de même signe dans cet ensemble, donc il suffit d'établir l'inégalité $\left|u_{n}(z)\right|>$ $\left|u(z)-u_{n}(z)\right|$. Pour cela, on observe que

$$
\left|u(z)-u_{n}(z)\right| \leq \sum_{p=n+1}^{+\infty} a_{p} \leq 2^{-n-1} c_{n} \varrho_{n}
$$

quel que soit $z$ en raison de $\left(4_{n}\right)$, et que $\left|u_{n}(z)\right| \geq 2^{-n} c_{n} \varrho_{n}$ pour tout $z \in$ $\Omega_{n}(\xi)$, en vertu de $\left(5_{n}\right)$. Il résulte de cette propriété que, pour tout $\xi \in K$, la restriction à toute demi-droite issue de $\xi$ de la fonction $\operatorname{sgn}(u)$ n'a pas de limite au point $\xi$. En effet, toute demi-droite issue de $\xi$ rencontre, dans tout voisinage de $\xi$, tous les ensembles $\Omega_{n}(\xi)$ d'indice assez grand, parce que la suite $\left(b_{n}\right)$ converge vers 1 et que la suite $\left(\varrho_{n}\right)$ converge vers 0 .

Nous allons maintenant prouver que le module de continuité $\sigma_{f}$ de la fonction $f$ vérifie, si la constante $\lambda$ est bien choisie, l'inégalité $\sigma_{f}(t) \leq \sigma(t / 2)$ pour tout $t>0$. Pour cela, nous utiliserons le

Lemme 2. Soit $\left(h_{n}\right)$ une suite de fonctions de classe $\mathcal{C}^{1}$, définies dans $\partial D$ et à valeurs réelles. On suppose qu'il existe deux suites $\left(d_{n}\right)$ et $\left(e_{n}\right)$ de nombres $\geq 0$ et une application décroissante $\tau:[0,+\infty[\rightarrow[0,+\infty[$ vérifiant les conditions suivantes:

(e) La suite $\left(e_{n}\right)$ est décroissante et on a, pour tout entier $n \geq 1$,

$$
\sup _{\xi \in \partial D}\left|h_{n}(\xi)\right| \leq e_{n} \quad \text { et } \quad \sum_{p=n}^{+\infty} e_{p} \leq 2 e_{n} .
$$

(d) Pour tout entier $n \geq 1$,

$$
\sup _{\xi \in \partial D}\left|h_{n}^{\prime}(\xi)\right| \leq d_{n} \quad \text { et } \quad d_{1}+\ldots+d_{n}+2 \leq \tau\left(2 e_{n}\right) .
$$

Alors la fonction $h=\sum_{n=1}^{+\infty} h_{n}$ possède la propriété de régularité suivante: pour tout $x^{\prime}$ tel que $\left|x^{\prime}\right| \leq 2 e_{1}$, on a

$$
\left|h\left(x+x^{\prime}\right)-h(x)\right| \leq\left|x^{\prime}\right| \tau\left(\left|x^{\prime}\right|\right) .
$$

Démonstration. Il suffit de prouver que, pour tout $x \in \partial D$, tout $n \geq 1$ et tout $x^{\prime}$ tel que $2 e_{n+1} \leq\left|x^{\prime}\right| \leq 2 e_{n}$, on a

$$
\left|h\left(x+x^{\prime}\right)-h(x)\right| \leq\left|x^{\prime}\right| \tau\left(\left|x^{\prime}\right|\right) .
$$

Pour cela, on écrit

$$
\begin{aligned}
\left|h\left(x+x^{\prime}\right)-h(x)\right| & \leq\left|x^{\prime}\right|\left(d_{1}+\ldots+d_{n}\right)+2 \sum_{p=n+1}^{+\infty} e_{p} \\
& \leq\left|x^{\prime}\right|\left(d_{1}+\ldots+d_{n}+2\right) \leq\left|x^{\prime}\right| \tau\left(2 e_{n}\right) \leq\left|x^{\prime}\right| \tau\left(\left|x^{\prime}\right|\right),
\end{aligned}
$$

d'où le résultat. 
Nous revenons à la démonstration du théorème. En vue d'appliquer le résultat précédent nous posons, pour tout entier $n \geq 1$, tout $\xi \in \partial D$ et tout $t \geq 0, h_{n}(\xi)=a_{n} \Re \varphi_{\alpha_{n}}\left(\xi^{k_{n}}\right), e_{n}=a_{n}, d_{n}=a_{n} k_{n}\left\|\varphi_{\alpha_{n}}^{\prime}\right\|_{\infty}$ et $\tau(t)=$ $\inf _{h \leq t} \sigma(h / 2) / h$. La condition (e) est satisfaite en raison des inégalités $\left(4_{n}\right)$, et la condition (d) l'est en raison des inégalités $\left(6_{n}\right)$; d'autre part, la fonction $\tau$ est évidemment décroissante. On prouve ainsi que

$$
\left|f\left(x+x^{\prime}\right)-f(x)\right| \leq\left|x^{\prime}\right| \tau\left(\left|x^{\prime}\right|\right) \leq \sigma\left(\left|x^{\prime}\right| / 2\right)
$$

pour tout $x$ et tout $x^{\prime}$ tel que $\left|x^{\prime}\right| \leq 2 a_{1}$. Par conséquent, il suffit de poser

$$
\lambda=\min \left(1, \frac{\sigma\left(a_{1}\right)}{2 \sum_{n=1}^{+\infty} a_{n}}\right)
$$

pour que la fonction $f$ définie par l'égalité $(7)$ vérifie l'inégalité $\sigma_{f}(t) \leq$ $\sigma(t / 2)$ pour tout $t>0$.

Retour au demi-plan. Nous identifions $\mathbb{R}^{2}$ et $\mathbb{C}$, et nous conservons les notations précédentes. Nous pouvons supposer que (par exemple) $1 \notin K$. Dans ce cas nous posons, pour tout $z \in \mathbb{C} \backslash\{-i\}$,

$$
T(z)=\frac{z-i}{z+i}
$$

puis $v(z)=u \circ T(z)$. La fonction $v$ ainsi définie est l'intégrale de Poisson dans le demi-espace de la fonction bornée $g$ définie dans $\mathbb{R}$ par $g=f \circ T$. Nous allons montrer que cette fonction $g$ et le compact $L \subset \mathbb{R}$ défini par $L=T^{-1}(K)$ possèdent les propriétés voulues.

On voit facilement que, pour tout $\left(\xi, \xi^{\prime}\right) \in \mathbb{R} \times \mathbb{R}$,

$$
\left|g(\xi)-g\left(\xi^{\prime}\right)\right| \leq \sigma_{f}\left(2\left|\xi-\xi^{\prime}\right|\right) \leq \sigma\left(\left|\xi-\xi^{\prime}\right|\right),
$$

donc la fonction $g$ possède la propriété de régularité requise. D'autre part, le compact $L$ vérifie $m(L)>0$ parce que $|K|>0$ et que la transformation $T$ conserve la mesure harmonique. Il nous reste donc à montrer que, en tout point $\xi \in L$, la fonction $\operatorname{sgn}(P(g))$ n'a pas de limite, dans les deux sens précisés par l'énoncé du théorème. Pour tout $\xi \in \mathbb{R}$, tout $a>0$ et tout $C>0$, nous désignons par $\Gamma_{a, C}(\xi)$ l'ensemble des points $z=x+i y \in \mathbb{R}_{+}^{2}$ tels que $|x-\xi|<a y$ et $|z-\xi| \leq C$. Comme la suite $\left(\varrho_{n}\right)$ converge vers 0 , et que la suite $\left(b_{n}\right)$ converge vers 1 , un argument de représentation conforme permet de voir que, pour tout $a>0$,

$$
T\left(\Gamma_{a, \varrho_{n}}(\xi)\right) \subset \Gamma_{b_{n}}(T(\xi))
$$

si $n$ est assez grand. Par suite, la restriction de la fonction $\operatorname{sgn}(P(g))=$ $\operatorname{sgn}(v)$ à toute droite issue du point $\xi$ n'a pas de limite, parce que la fonction $\operatorname{sgn}(P(f))=\operatorname{sgn}(u)$ a, comme on l'a vu, la propriété analogue relativement à toute droite issue du point $T(\xi)$. 
Montrons enfin que, en moyenne par rapport à toute mesure finie $\mu$ telle que $\mu\left(\mathbb{R}_{+}^{2}\right) \neq 0$, la fonction $\operatorname{sgn}(P(g))$ n'a pas de limite en tout point $\xi \in L$. On peut évidemment supposer que $\mu\left(\mathbb{R}_{+}^{2}\right)=1$, et dans ce cas nous pouvons trouver $a>0$ et $C>0$ vérifiant les deux conditions

$$
\mu\left(\Gamma_{a, C}(0)\right) \geq 3 / 4 \text { et }|\mu|\left(\left(\Gamma_{a, C}(0)\right)^{\mathrm{c}}\right) \leq 1 / 4 .
$$

Ayant fixé un point $\xi \in L$, on peut trouver une constante $C^{\prime}>0$ telle que $C^{\prime}|z-\xi| \leq|T(z)-T(\xi)| \leq 2|z-\xi|$ pour tout $z \in \mathbb{R}_{+}^{2}$ tel que $|z| \leq C$. Nous posons $b=\operatorname{sgn}(P(g))$; pour montrer que l'intégrale

$$
I(\varepsilon)=\int_{\mathbb{R}_{+}^{2}} b(\xi+\varepsilon z) \mu(d z)
$$

n'a pas de limite quand $\varepsilon$ tend vers 0 , nous montrerons que la suite $\left(I\left(\varrho_{n} / 2 C\right)\right)$ est divergente. Pour cela nous écrivons, dès que $2^{-n} \leq C C^{\prime}$,

$$
\begin{aligned}
I\left(\varrho_{n} /(2 C)\right)= & \int_{\Gamma_{a, C}(0) \cap\left(D\left(0,2^{-n} / C^{\prime}\right)\right)^{\mathrm{c}}} b\left(\xi+\varrho_{n} z /(2 C)\right) \mu(d z) \\
& +\int_{\Gamma_{a, C}(0) \cap D\left(0,2^{-n} / C^{\prime}\right)} b\left(\xi+\varrho_{n} z /(2 C)\right) \mu(d z) \\
& +\int_{\left(\Gamma_{a, C}(0)\right)^{\mathrm{c}}} b\left(\xi+\varrho_{n} z /(2 C)\right) \mu(d z) .
\end{aligned}
$$

En raison du choix des constantes $C$ et $C^{\prime}$ on a, pour tout entier $n \geq 1$ et tout $z \in \Gamma_{a, C}(0) \cap\left(D\left(0,2^{-n} / C^{\prime}\right)\right)^{\mathrm{c}}, 2^{-n} \varrho_{n} \leq\left|T\left(\xi+\varrho_{n} z /(2 C)\right)-T(\xi)\right| \leq \varrho_{n}$. En utilisant cette propriété et l'inclusion (8) on voit que, pour tout $n$ assez grand, $T\left(\xi+\varrho_{n} z /(2 C)\right) \in \Omega_{n}(T(\xi))$ (et donc $b\left(\xi+\varrho_{n} z /(2 C)\right)=(-1)^{n}$ ) pour tout $z \in \Gamma_{a, C}(0) \cap\left(D\left(0,2^{-n} / C^{\prime}\right)\right)^{\mathrm{c}}$. On en déduit facilement, en utilisant l'égalité (10) et les conditions (9), que $I\left(\varrho_{n} /(2 C)\right) \geq 1 / 4$ pour tout $n$ pair assez grand, alors que $I\left(\varrho_{n} /(2 C)\right) \leq-1 / 4$ pour tout $n$ impair assez grand. Ceci termine la démonstration.

Remarque. Dans la perspective d'une extension du théorème en dimension supérieure, il nous paraît intéressant de signaler une relation entre le "problème du signe" étudié dans le présent article et un des problèmes ouverts concernant les gradients harmoniques ([3]). Dans ce but, les quelques observations qui suivent nous seront utiles.

Notons $E_{n}$ l'ensemble des fonctions $f$ définies dans $\mathbb{R}^{n}$ telles que la fonction $\operatorname{sgn}(P(f))$ vérifie la propriété $L^{\prime}(\xi)$ pour presque tout $\xi \in \mathbb{R}^{n}$, et $F_{n}$ l'ensemble des fonctions $f$ définies dans $\mathbb{R}^{n}$ telles que $\nabla P(f)$ admette une limite non-tangentielle presque partout dans $\mathbb{R}^{n}$. Le théorème prouvé dans cet article montre que, si $n=1$, on a l'équivalence

$$
\operatorname{Lip}_{\alpha}\left(\mathbb{R}^{n}\right) \subset E_{n} \Leftrightarrow \alpha=1 .
$$


D'autre part nous avons aussi, si $n=1$, l'équivalence

$$
\operatorname{Lip}_{\alpha}\left(\mathbb{R}^{n}\right) \subset F_{n} \Leftrightarrow \alpha=1 .
$$

En effet l'inclusion $\operatorname{Lip}_{1}(\mathbb{R}) \subset F_{1}$ a été notée au cours de la preuve de l'assertion (i) du théorème. D'autre part, on peut trouver $f \in \bigcap_{\alpha<1} \operatorname{Lip}_{\alpha}(\mathbb{R})$ telle que $\nabla P(f)$ n'ait pas de limite non-tangentielle presque partout. Pour cela, on transporte le problème dans le disque unité $D$ du plan complexe et on utilise les trois propriétés connues suivantes : la première est l'existence d'une fonction de Bloch définie dans $D$ qui n'admet pas de limite nontangentielle presque partout. La deuxième est le fait que la fonction $z \mapsto$ $\sum_{n=0}^{+\infty} a_{n} z^{n}$ est une fonction de Bloch si et seulement si la fonction $t \mapsto$ $\sum_{n=1}^{+\infty}\left(a_{n} / n\right) e^{i n t}$ appartient à la classe de Zygmund $\Lambda^{*}$ relative au cercle unité. La troisième est l'inclusion $\Lambda^{*} \subset \bigcap_{\alpha<1} \operatorname{Lip}_{\alpha}$.

Ces deux équivalences montrent que l' "argument du gradient" utilisé dans la preuve de l'assertion (i) du théorème est en un certain sens optimal, et que l'appartenance à $E_{1}$ et l'appartenance à $F_{1}$ requièrent essentiellement la même régularité.

D'autre part, nous observons que l'équivalence (12) est vraie en toute dimension. En effet, si $f \in \operatorname{Lip}_{1}\left(\mathbb{R}^{n}\right)$, les $n$ dérivées partielles $\partial f / \partial \xi_{i}$ sont bornées dans $\mathbb{R}^{n}$, et par suite les $n$ dérivées partielles $\partial P(f) / \partial x_{i}$ le sont dans $\mathbb{R}_{+}^{n+1}$. Pour cette raison, elles ont une limite non-tangentielle en presque tout point de la frontière. En ce qui concerne la dernière dérivée partielle, nous écrivons

$$
\frac{\partial P(f)}{\partial y}=-\sum_{j=1}^{n} \frac{\partial P\left(R_{j}(f)\right)}{\partial x_{j}}=-\sum_{j=1}^{n} P\left(\frac{\partial R_{j}(f)}{\partial \xi_{j}}\right)=-\sum_{j=1}^{n} P\left(R_{j}\left(\frac{\partial f}{\partial \xi_{j}}\right)\right),
$$

où les $R_{j}$ sont les transformations de Riesz. Comme ces transformations appliquent $L^{\infty}$ dans BMO et que l'intégrale de Poisson d'une fonction de BMO a une limite non-tangentielle presque partout, on voit finalement que $\nabla P(f)$ admet une limite non-tangentielle presque partout, et donc que $f \in F_{n}$. Réciproquement, si $\alpha<1$, il existe (comme nous l'avons vu précédemment) une fonction $f \in \operatorname{Lip}_{\alpha}(\mathbb{R})$ et un borélien $B \subset \mathbb{R}$, de mesure de Lebesgue $>0$, tels que $\nabla P(f)$ n'admette de limite non-tangentielle en aucun point de $B$. Nous définissons alors une application $\tilde{f}: \mathbb{R}^{n} \rightarrow \mathbb{R}$ en posant $\widetilde{f}(x)=f\left(x_{1}\right)$. Il est clair que $\tilde{f} \in \operatorname{Lip}_{\alpha}\left(\mathbb{R}^{n}\right)$, et que

$$
P(\widetilde{f})(x, y)=P(f)\left(x_{1}, y\right)
$$

pour tout $(x, y) \in \mathbb{R}_{+}^{n+1}$. On en déduit que $\nabla P(\widetilde{f})$ n'a de limite nontangentielle en aucun point de $B \times \mathbb{R}^{n-1}$, ce qui prouve que $\widetilde{f} \notin F_{n}$.

La démonstration de (i) utilise successivement deux arguments. Le premier est l'implication $\alpha=1 \Rightarrow \operatorname{Lip}_{\alpha}(\mathbb{R}) \subset F_{1}$, qui est vraie en toute dimension, comme nous venons de le voir. Le deuxième argument est basé sur le 
fait suivant, élémentaire mais crucial : si $f$ est une fonction non constante sur $\mathbb{R}$ et si $f \in \operatorname{Lip}_{1}(\mathbb{R})$, alors la limite non-tangentielle de $\nabla P(f)$ est non nulle presque partout.

Cette propriété est en défaut en dimension supérieure, car on sait ([3]) qu'il existe $\varepsilon>0$ et $f \in \mathcal{C}^{1+\varepsilon}\left(\mathbb{R}^{2}\right)$ tels que $\nabla P(f)$ s'annule sur un ensemble de mesure $>0$ du bord du demi-espace $\mathbb{R}_{+}^{3}$, sans que $f$ soit constante. La question de savoir si ce phénomène disparaît lorsque $f \in \mathcal{C}^{\alpha}\left(\mathbb{R}^{2}\right)$ avec $\alpha$ assez grand ou $\alpha=+\infty$ est un problème ouvert. Il est tentant d'imaginer qu'il existe, pour toute valeur de la dimension $n$, un "niveau de régularité" $\alpha_{n}$ tel que, pour tout $\alpha \geq \alpha_{n}$ et toute fonction non constante $f \in \mathcal{C}^{\alpha}\left(\mathbb{R}^{n}\right), \nabla P(f)$ ne peut s'annuler sur un ensemble de mesure $>0$ du bord. Si tel était le cas, les observations précédentes permettraient d'en déduire que $\mathcal{C}^{\alpha}\left(\mathbb{R}^{n}\right) \subset E_{n}$ pour tout $\alpha \geq \alpha_{n}$. Inversement, une réponse négative au problème du signe en dimension $n$ pour une fonction $f \in \mathcal{C}^{\alpha}\left(\mathbb{R}^{n}\right)$ permettrait évidemment d'affirmer que l'hypothétique nombre $\alpha_{n}$ est $>\alpha$.

\section{Références}

[1] L. Chevalier, Une "formule de Tanaka" en analyse harmonique et quelques applications, Adv. in Math. 138 (1998), 182-210.

[2] L. Chevalier et A. Dufresnoy, Densité de l'intégrale d'aire et intégrales singulières, Ark. Mat. 38 (2000), 209-221.

[3] T. H. Wolff, Counterexamples with harmonic gradients in $\mathbb{R}^{3}$, in: Essays on Fourier Analysis in Honor of Elias M. Stein, Princeton Univ. Press, Princeton, NJ, 1995, $321-384$.

Institut Fourier

U.M.R. 5582 C.N.R.S./U.J.F.

B.P. 74

38402 Saint Martin d'Hères, France

E-mail: lucchev@fourier.ujf-grenoble.fr dufresn@fourier.ujf-grenoble.fr 